\title{
Modeling Macro-Sized, High Aspect Ratio Through-Hole Filling by Multi-Component Additive-Assisted Copper Electrodeposition
}

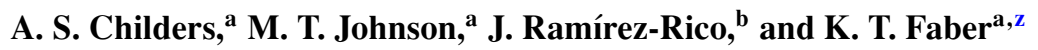 \\ ${ }^{a}$ Department of Materials Science and Engineering, Robert R. McCormick School of Engineering and Applied Science, \\ Northwestern University, Evanston, Illinois 60208, USA \\ ${ }^{b}$ Departmento Física de la Materia Condensada-ICMS, Universidad de Sevilla-CSIC, 41012 Sevilla, Spain
}

\begin{abstract}
A multi-element, time-dependent model is used to examine additive-assisted copper electroplating in macro-channels. This model is an adaptation of the work of Akolkar and Landau [J. Electrochem. Soc., 156, D351 (2009)], used to describe plating in micro-vias for integrated circuits. Using their method for describing species movement in the channel, the model has been expanded to include transport and adsorption limitations of the inhibitor and accelerator, as well as the copper ions in solution. The model is used to investigate copper plating as an infiltration method across many size scales and aspect ratios. Biomorphic graphite scaffolds produced from wood are used as a representative system and the results of a two-additive bath are used to characterize the behavior of the additives and determine the effectiveness of the plating. The results indicate that at macro-scales, channel dimensions play an increasingly important role in dictating the behavior of additive-assisted plating. Because additive systems are designed to establish differential surface coverage within the channel, the success of which is determined by the additive's rates of diffusion and adsorption, certain size scale/aspect ratio combinations preclude such coverage. A guide for sample geometries that may be successfully infiltrated with a two-additive bath is provided.

(C) 2013 The Electrochemical Society. [DOI: 10.1149/2.018312jes] All rights reserved.
\end{abstract}

Manuscript submitted June 6, 2013; revised manuscript received August 14, 2013. Published August 29, 2013. This paper is part of the JES Focus Issue on Electrochemical Processing for Interconnects.

Electrodeposition is a common method of producing multicomponent material systems. ${ }^{1,2}$ This technique is useful in overcoming common melt-based composite processing constraints, such as high melting points, fragile scaffolds, non-wetting components, and significant coefficient of thermal expansion (CTE) mismatch. Unlike melt infiltration or reactive wetting, electrodeposition is a low-temperature, non-reactive process. While electroformed copper is employed in the fabrication of items such as musical instruments, reflectors, and heat exchangers, ${ }^{1}$ the electronics sector is responsible for the majority of advancements in the field, especially in terms of use as an infiltration method. Electrodeposition is used for interconnect metallization in integrated circuits, where small channels in a silicon wafer, known as vias, are filled with high purity copper. IBM pioneered this technique in 1998 to replace aluminum vapor deposition. ${ }^{3,4}$ While the microvias used in current integrated circuit technology can reach several hundred microns in dimension, channel size is trending toward nanosized, closely packed channels. ${ }^{5-7}$ In other functional composites, one might contend with larger length scales and aspect ratios compared to metal interconnects. With this as motivation, we explore the feasibility of using existing electroplating techniques for composite processing of non-interconnect systems through a multi-component, time-dependent model. Specifically, we investigate infiltration of a metal into highly porous ceramic scaffolds with macro-sized channels.

As a macro-porous system for study, we examine biomorphic SiCand graphite- $\mathrm{Cu}$ composites produced by electrodeposition, which may have possible applications in thermal management systems. ${ }^{8,9}$ In these materials, wood is used as a porous precursor, which, after pyrolysis, results in a ceramic that retains the naturally-optimized cellular structure of the parent material. The wood species of the scaffold precursor determines the pore size, volume fraction, distribution, and connectivity, offering a wide range of possible materials and tailorable porous systems. Biomorphic graphite and $\mathrm{SiC}$, as well as other woodbased ceramics and composites, are popular options for high-porosity materials due to their relative ease of processing compared to traditional porous ceramic-processing methods. ${ }^{10-13}$ Figure 1 shows an example of biomorphic graphite, produced from a red oak (Quercus rubra) precursor, highlighting the anisotropic porous geometry. This highly connected porosity is a result of the vascular system in the tree,

${ }^{\text {z} E-m a i l: ~ k-f a b e r @ n o r t h w e s t e r n . e d u ~}$ which contains channels that extend over several meters. ${ }^{14-16}$ Depending on the species of the wood precursor, these channels can range in diameter from 8 to $200 \mu \mathrm{m}$ in the graphitized form. In laboratoryscale graphitized scaffolds, this translates to channels with aspect ratios $(\mathrm{AR}=$ length $(\mathrm{L}) /$ diameter $(2 \mathrm{R})$ ) ranging from roughly 15 to 375. This example reveals several common processing challenges, such as non-uniform pore size distribution, fragile scaffolds, and high aspect ratio channels.

Copper is commonly plated from an acidic electrolyte bath containing common families of additives, such as inhibitors (e.g. poly(ethylene glycol) (PEG)) and accelerators (e.g. bis(3-sulfopropyl) disulfide (SPS) or its monomer, 3-mercapto-1-propanesulfonate (MPS)). ${ }^{17,18}$ These additive systems are designed to adsorb to the inner sidewalls of the channels in such a way that plating is promoted near the bottom of the via in a blind-hole, or the middle of the channel in a through-hole, while obstructing plating near the mouth, resulting in void-free copper plating known as super-conformal growth. ${ }^{19,20}$ Without such systems, incomplete growth (voids, seams, etc.) and even total choke-off of the channel is likely. X-ray computed tomography has been used to study the initial stages of copper plating in biomorphic graphite, the results of which are shown in Figure $2 .{ }^{21}$ The substrate experienced extensive surface plating that effectively blocked the channels' mouths and prevented further interior plating. An example of excessive choke-off is seen in the micrograph in Figure 3, indicative that choke-off is a crucial consideration in composite processing of this nature. Therefore, the ability to choose an electrolyte bath and additive system that prevents this phenomenon is desired. Because most reported electrolyte baths evaluated for use in infiltration have been designed for interconnect metallization (axial length, $L \sim 0.1-10 \mu \mathrm{m}$ ), extrapolating optimized plating conditions for alternative length scales, such as those seen in biomorphic graphite scaffolds, is often done by trial and error. However, because of the large number of controllable experimental factors involved in electroplating, experimentally determining all parameters and plating conditions for an optimized electrolyte bath quickly becomes unmanageable. Therefore, filling models have been developed to predict plating behavior and guide experimental choices. ${ }^{4,22-35}$ While there are many available approaches to and techniques for modeling copper electroplating in channels, a descriptive, multi-component approach developed by Akolkar and Landau has been used in this work due to its ease of interpretation and implementation. ${ }^{19}$ The additive distribution is dictated by the transport and adsorption limitations of the additive 


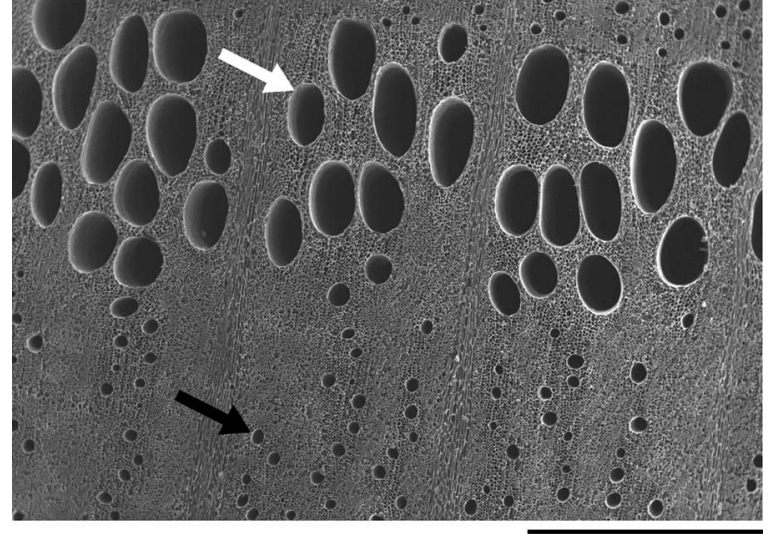

$1 \mathrm{~mm}$

Figure 1. Example micrograph of porous biomorphic graphite derived from red oak. The vascular channels that extend over large distances (several meters) in the tree become the highly connected pore network in the ceramic scaffold. Therefore, both the channels derived from the early-growth wood (white arrow) and late-growth wood (black arrow) span the entire length of the sample specimen.

molecules in the bath. Their approach retains the details necessary for accurate predictions without relying on empirical parameters, and without requiring the use of finite element analysis or dedicated electrochemical software. ${ }^{34,35}$ While this useful tool is straightforward and easy to implement, its dependence on a few simplifying assumptions limits its broader applicability. The model proves to be inaccurate at large channel dimensions; most notable is its inability to predict observed sub-conformal growth at non-optimized conditions. Specifically, the defined additive characteristics that determine catalytic surface coverage apply only over small length scales. As the aspect ratio of a channel increases, so does the likelihood of choke-off, as plating at the mouth can easily obstruct the channel opening before the inner volume of the channel is filled.

Therefore, the ability to predict this phenomenon is a necessary aspect of any model that is to be applicable in all situations. This paper



Figure 2. Example 3D reconstruction of XCT on initial plating in a biomorphic graphite scaffold, illustrating the extensive surface plating and choke-off that can occur. ${ }^{21}$



Figure 3. SEM micrograph of a transverse cross section of plated channels in a biomorphic scaffold produced from red oak that has experienced early chokeoff at the channel mouths. The unplated interior of the channel is indicated by the white bracket, while the blocked channel mouths are indicated by black arrows.

builds on the work of Akolkar and Landau by utilizing their descriptions of bath species evolution to produce a model that is adaptable over many length scales and thus, of use to researchers in composite processing. This is accomplished by three specific additions to their model: i) imposing transport limitations on both the accelerator and copper ions in the bath, as opposed to only the inhibitor, ii) forcing consumptive plating constraints on the copper ions, and iii) implementing appropriate boundary and initial conditions that do not depend on initial knowledge of sample geometry or additive availability. This revised model is used to characterize the effectiveness of additive-assisted plating systems in macro-sized channels, such as those seen in porous biomorphic scaffolds.

\section{The Additives-Assisted Electrodeposition Model}

In this work, copper is plated from a $0.88 \mathrm{M} \mathrm{CuSO}_{4} .5 \mathrm{H}_{2} \mathrm{O}$ solution prepared with $0.54 \mathrm{M} \mathrm{H}_{2} \mathrm{SO}_{4}, 60 \mathrm{ppm}$ chloride (from $\mathrm{HCl}$ ) and a balance of deionized water. Both an inhibitor (PEG) and an accelerator (SPS) are used to conform to the industrial plating standard of integrated circuits and printed circuit boards. ${ }^{17,36}$ A magnetic stir plate was used to maintain constant agitation throughout plating, and the sample was oriented such that bath flow was perpendicular to the channel axis to minimize internal flow due to electrolyte movement. Therefore, forced convective effects are not considered in this work but have been addressed elsewhere. ${ }^{24}$

Inhibitors prevent plating by forming a thin, passivating film at the substrate surface that obstructs and envelopes the copper ions. ${ }^{37-39}$ The inhibiting capability is dependent on the molecular weight of the polymer, and has the ability to reduce deposition kinetics by as much as two orders of magnitude. ${ }^{40}$ Therefore, a sufficiently large cathodic potential is required for copper ion reduction. Inhibitors diffuse slowly $\left(D_{\mathrm{PEG}}=5 \times 10^{-7} \mathrm{~cm}^{2} / \mathrm{s}\right.$ ), and bond weakly, but quickly, to the substrate surface. ${ }^{41}$ Because of this weak interaction, inhibitors can only be adsorbed on unoccupied substrate sites where the energetic barrier to bonding is low.

Conversely, accelerators bond strongly to the substrate surface and can therefore adsorb to either a free site or an inhibitor occupied site (i.e. replacement), reactions which occur more slowly than the inhibitor adsorption reaction. ${ }^{40}$ However, accelerator molecules diffuse up to two orders of magnitude faster than inhibitors (e.g. $D_{\text {SPS }}=5$ $\left.\times 10^{-5} \mathrm{~cm}^{2} / \mathrm{s}\right) .{ }^{41}$ The accelerator increases the plating reaction kinetics by disrupting the film created by the inhibitor, increasing site availability for easy reduction of copper ions.

The additive-assisted method of infiltration has been designed for the micro-via system such that the inherent diffusion rates, adsorption rates, and bonding strengths of the additives leads to a favorable distribution of the compounds on the inner sidewalls of the 
channel. Inhibitor coverage is limited to the outer end of the channel due to its transport limitations (slow diffusion) and consumptive adsorption (quick bonding). Accelerator coverage is more prominent at the channel's center as the molecules that diffuse rapidly and form bonds slowly "out-run" the inhibitor molecules and avoid consumptive adsorption. This area of heightened accelerator coverage will eventually expand outwards as the accelerator molecules slowly displace the inhibitor as plating progresses. In an ideal system, this competitive adsorption for sites on the substrate results in increased deposition kinetics at the channel middle due to the presence of the accelerator, and suppressed deposition kinetics at the channel opening due to the adsorbed inhibitor. In the present consideration, only axial diffusion into the channel (along the z-axis) is considered, since the effects of radial diffusion limitations will be negligible in high aspect ratio pores. Additionally, all substrate sites are assumed equivalent (number of free sites is equal to $(1-\theta)$, where $\theta$ is the fraction of total surface coverage of the additive species), adsorbed molecules do not interact, and adsorption cannot proceed beyond monolayer coverage. This model assumes that adsorbed molecules may only be displaced by a stronger bonding available species in solution. Experimental observations justify this assumption considering the high percentage of equilibrium surface coverage of the additives. ${ }^{42}$ Finally, the additives in the model are not consumed or deactivated throughout the plating process; many investigations find only negligible levels of included sulfur, chlorine, and carbon, indicating levels of incorporation less than $100 \mathrm{ppm}^{43}$

By accounting for the possible fluxes of additives into and out of solution in a channel, a set of equations that quantitatively describes the additive coverage on the channel wall as a function of time and position may be developed. The treatment provided by Akolkar and Landau for use in vias considers the evolution of inhibitor and accelerator additives and imposes transport limitations only on inhibitor molecules. ${ }^{19}$ This is depicted in the schematic of a channel in Figure 4a. However, as described above, a robust treatment of the problem must also account for the transport and adsorption limitations of accelerator molecules and copper ions and reflect that the initial simplifying assumptions may not hold in all cases. This scheme is depicted in Figure 4b. Although Akolkar and Landau have provided a detailed development of the approach, we include only the most salient equations that form the basis of the present work. The change

a)
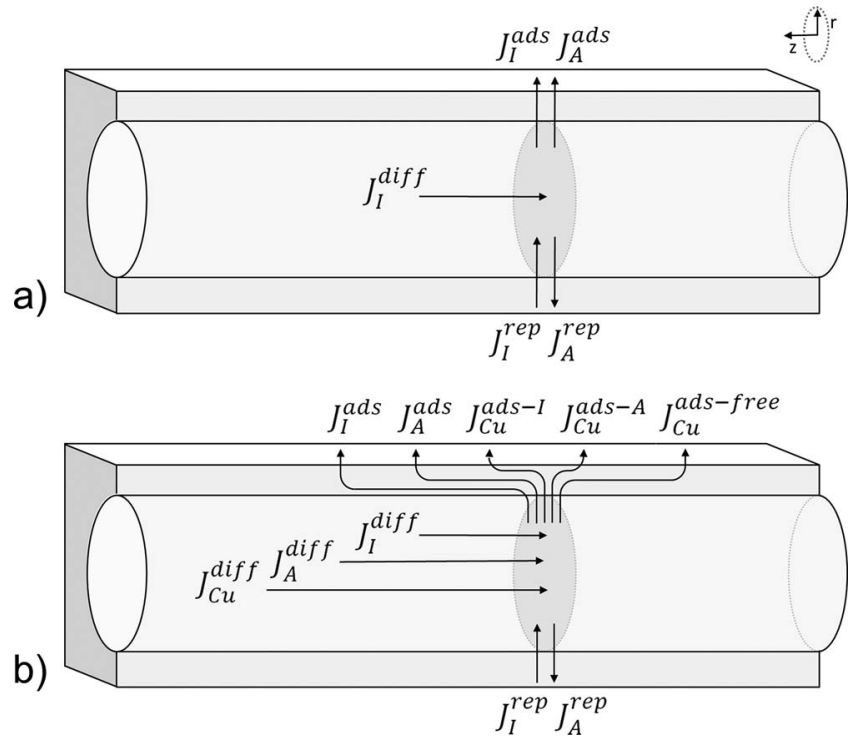

Figure 4. Diagram of the accounted movements of bath species for two approaches: a) the method implemented by Akolkar and Landau in which only the inhibitor molecule is limited by diffusion ${ }^{19}$ and b) the proposed system, in which all bath species required for plating, including the inhibitor, accelerator, and copper ions, are described fully by transport $\left(J^{\text {diff }}\right)$ and adsorption $\left(J^{a d s}\right)$ limitations. in inhibitor concentration over time is

$$
\frac{\partial C_{I}}{\partial t}=D_{I} \frac{\partial^{2} C_{I}}{\partial z^{2}}-\frac{2}{R}\left[k_{I} C_{I}\left(1-\theta_{I}-\theta_{A}\right)-k_{\text {rep }} C_{A} \theta_{I}\right]
$$

while the evolution of inhibitor surface coverage is described by

$$
\frac{\partial \theta_{I}}{\partial t}=\frac{1}{\Gamma_{I}}\left[k_{I} C_{I}\left(1-\theta_{I}-\theta_{A}\right)-k_{r e p} C_{A} \theta_{I}\right]
$$

where $D_{I}, C_{I}$, and $k_{I}$ are the diffusion coefficient, local concentration, and rate constant for adsorption on a free site of the inhibitor species, respectively, and $\theta_{I}$ and $\theta_{A}$ are the fractional surface coverage of the substrate channel wall for both the inhibitor and accelerator, respectively. The surface saturation concentration of the inhibitor molecule, $\Gamma_{I}$, accounts for the difference in size between a typical inhibitor molecule and an accelerator molecule a ratio of the $\Gamma$-terms for the two species nominally describes the number of accelerator molecules that can adsorb to an area previously occupied by one inhibitor molecule. Because an accelerator molecule may displace an absorbed inhibitor molecule, there is a flux of inhibitor molecules reintroduced into the solution, where $k_{\text {rep }}$ is the rate constant for a replacement reaction and $C_{A}$ is the concentration of the accelerator. The radius of the channel, $R$, decreases as plating progresses. The first term on the right side of Eq. 1 is associated with the Fickian diffusion limitations of the molecules. In both Eqs. 1 and 2, the two contributions in the bracketed terms represent the possible movement of the inhibitor - adsorption of the species on the channel wall (first, $J^{a d s}$ ) and replacement of the more weakly bonding inhibitor molecule by the more strongly bonding accelerator molecule (second, $J^{\text {rep }}$ ).

A similar approach based on the derivations for the inhibitor by Akolkar and Landau was applied to the evolution of the accelerator. ${ }^{19}$ The assumption of bulk availability of the accelerator at all locations becomes increasingly unrealistic as the dimensions of the channel are increased. The resulting change in accelerator concentration over time is

$$
\frac{\partial C_{A}}{\partial t}=D_{A} \frac{\partial^{2} C_{A}}{\partial z^{2}}-\frac{2}{R}\left[k_{A} C_{A}\left(1-\theta_{I}-\theta_{A}\right)+k_{r e p} C_{A} \theta_{I}\right],
$$

while the evolution of accelerator surface coverage is described by

$$
\frac{\partial \theta_{A}}{\partial t}=\frac{1}{\Gamma_{A}} k_{A} C_{A}\left(1-\theta_{I}-\theta_{A}\right)+\frac{1}{\Gamma_{\mathrm{I}}} k_{r e p} C_{A} \theta_{I}
$$

where $D_{A}, k_{A}$, and $\Gamma_{A}$ are the diffusion coefficient, rate constant for adsorption on a free site, and saturation surface concentration of the accelerator species, respectively. The sign on the term associated with the replacement reaction in both equations is opposite of that in the equation describing the inhibitor evolution, indicating that unlike the inhibitor molecule, an accelerator molecule may initiate replacement reactions but its strong bond is irreversible.

At micro-length scales, it was only necessary for Akolkar and Landau to consider the evolution of additive species in the bath. ${ }^{19}$ However, like the addition of a transport limitation term for the accelerator, if this model is to be applicable over many length scales, a description of the final reactant in the bath is necessary. This approach also has been shown to be beneficial in other work. ${ }^{33-35}$ By treating the copper ions in solution as a third "additive" in the electrolyte, a treatment similar to those described above is applied. For example, $\mathrm{Cu}^{2+}$ ions, at $25^{\circ} \mathrm{C}$ in a $0.54 \mathrm{M}$ solutions of sulfuric acid, diffuse at a moderate rate $\left(D_{C u}=4.5 \times 10^{-6} \mathrm{~cm}^{2} / \mathrm{s}\right)$, and will have a Fickian flux term similar to Eqs. 1 and $3 .{ }^{44}$ Copper ions leave the solution when they are reduced and plated on the substrate walls, analogous to the adsorption of an additive. Electroplating rates are governed by Faraday's law of electrolysis:

$$
\frac{h}{t}=\frac{M_{C u}}{F \rho_{C u} n} i
$$

where $h$ is plated thickness, $M_{C u}$ is the molecular weight of copper, $F$ is Faraday's constant, $\rho_{C u}$ is the density of copper, $n$ is the number of electrons transferred in the reduction reaction, and $i$ is the current 


\begin{tabular}{|c|c|c|c|}
\hline Criterion & Value & Units & Source \\
\hline \multicolumn{4}{|l|}{ Constants } \\
\hline $\mathrm{F}$ & 96,485 & $\mathrm{C} / \mathrm{mol}$ & \\
\hline $\mathrm{R}_{\mathrm{g}}$ & 8.314 & $\mathrm{~J} / \mathrm{molK}$ & \\
\hline $\mathrm{M}_{\mathrm{Cu}}$ & 63.55 & $\mathrm{~g} / \mathrm{mol}$ & \\
\hline$\rho_{\mathrm{Cu}}$ & 8.94 & $\mathrm{~g} / \mathrm{cm}^{3}$ & \\
\hline $\mathrm{n}$ & 2 & - & \\
\hline \multicolumn{4}{|c|}{ Experimental Parameters } \\
\hline $\mathrm{L}$ & 0.3 & $\mathrm{~cm}$ & \\
\hline $\mathrm{R}$ & 0.003 & $\mathrm{~cm}$ & \\
\hline $\mathrm{C}_{\mathrm{Cu}}^{\text {bulk }}$ & 0.88 & $\mathrm{~mol} / \mathrm{cm}^{3}$ & \\
\hline$\eta$ & 0.19 & V & \\
\hline $\mathrm{T}$ & 293 & K & \\
\hline \multicolumn{4}{|c|}{ Additive Properties } \\
\hline $\mathrm{k}_{\mathrm{I}}$ & 0.1 & $\mathrm{~cm} / \mathrm{s}$ & 19,41 \\
\hline $\mathrm{k}_{\mathrm{A}}$ & 0.001 & $\mathrm{~cm} / \mathrm{s}$ & 19,41 \\
\hline $\mathrm{k}_{\mathrm{rep}}$ & $5 \times 10^{-6}$ & $\mathrm{~cm} / \mathrm{s}$ & 19,41 \\
\hline$\Gamma_{\mathrm{I}}$ & $6 \times 10^{-11}$ & $\mathrm{~mol} / \mathrm{cm}^{2}$ & 42 \\
\hline$\Gamma_{\mathrm{A}}$ & $8 \times 10^{-10}$ & $\mathrm{~mol} / \mathrm{cm}^{2}$ & 42 \\
\hline $\mathrm{D}_{\mathrm{I}}$ & $5 \times 10^{-7}$ & $\mathrm{~cm}^{2} / \mathrm{s}$ & 19,41 \\
\hline $\mathrm{D}_{\mathrm{A}}$ & $5 \times 10^{-5}$ & $\mathrm{~cm}^{2} / \mathrm{s}$ & 19,41 \\
\hline $\mathrm{D}_{\mathrm{Cu}}$ & $4.5 \times 10^{-6}$ & $\mathrm{~cm}^{2} / \mathrm{s}$ & 44 \\
\hline$\alpha_{\mathrm{I}}$ & 0.5 & - & 36 \\
\hline$\alpha_{\mathrm{A}}$ & 0.4 & - & 36 \\
\hline$\alpha_{\text {free }}$ & 0.5 & - & 19 \\
\hline $\mathrm{m}_{\mathrm{I}}$ & 0.6 & $\mathrm{cmA} / \mathrm{mol}$ & $36,40,49,50$ \\
\hline $\mathrm{m}_{\mathrm{A}}$ & 7.6 & $\mathrm{cmA} / \mathrm{mol}$ & $36,40,41$ \\
\hline $\mathrm{m}_{\text {free }}$ & 4.4 & $\mathrm{cmA} / \mathrm{mol}$ & $19,36,49,51-54$ \\
\hline
\end{tabular}

density. The current density in the Tafel regime of an electrochemical cell at the cathode is described by

$$
i=i_{o} \exp \left(-\frac{\alpha F \eta}{R_{g} T}\right)
$$

where $i_{o}$ is the exchange current density at the electrode surface, $\alpha$ is the cathodic transfer coefficient of copper deposition, and $\eta$ is the activation overpotential. An ion can plate either on an inhibitor occupied site, an accelerator occupied site, or a free site on the substrate, each process associated with unique plating kinetics. Assuming constant electrochemical parameters, the plating rate described by Eq. 5 is determined by the exchange current density term seen in Eq. 6, and will be different for each of the three types of sites. Given that the exchange current density scales linearly with the concentration of the reacting species, $i_{o}$ can be rewritten as $i_{o}=m C_{C u}$, where $m$ is a proportionality constant determined from cited literature values and noted in Table I. This substitution in Eq. 6 indicates a direct relationship between copper ion concentration and deposition kinetics for plating within the channel. The combination of Eqs. 5 and 6 describes the rate at which copper ions are removed from solution due to plating on each type of site. Therefore, the flux of copper ions leaving solution due to plating ("adsorption") is described by

$$
J_{C u}^{a d s}=\left[\frac{M_{C u}}{F \rho_{C u} n} m C_{C u} \exp \left(-\frac{\alpha F \eta}{R_{g} T}\right)\right] C_{C u} \theta
$$

where $m$ is the proportionality constant of the relationship between exchange current density and copper ion concentration for each of the three plating cases, and $\theta$ represents the surface fraction of substrate sites for the given conditions $\left(\theta_{I}\right.$ or $\theta_{A}$ for plating in the presence of an inhibitor or accelerator, respectively, or $\left(1-\theta_{I}-\theta_{A}\right)$ for plating on an unoccupied site). Faraday's law, the bracketed term above, dictates the plating rate and is analogous to the rate constant, $k$, which describes the adsorption of the inhibitor and accelerator species in Eqs. 1-4, and will be denoted as $k_{C u}{ }^{I}, k_{C u}{ }^{A}$, and $k_{C u}$ free for plating on an inhibitor occupied site, an accelerator occupied site, or a free site, respectively.
Therefore, the copper ion concentration evolution can be described by:

$$
\begin{aligned}
\frac{\partial C_{C u}}{\partial t}= & D_{C u} \frac{\partial^{2} C_{C u}}{\partial z^{2}}-\frac{2}{R}\left[k_{C u}^{I} C_{C u} \theta_{I}+k_{C u}^{A} C_{C u} \theta_{A}\right. \\
& \left.+k_{C u}^{f r e e} C_{C u}\left(1-\theta_{I}-\theta_{A}\right)\right]
\end{aligned}
$$

Eqs. 1-4 and 8 fully describe the time and location dependent evolution of a species present in an electrolyte in a channel and on the surface of the channel walls. In the current study, biomorphic samples with porosity extended through the entire thickness are considered. At the open ends of the channels, the species are present in their bulk concentrations $(\mathrm{C})$ :

$$
\text { At } z=0 \text { and } z=L \quad C_{I}=C_{I}^{b u l k}, C_{A}=C_{A}^{b u l k}, C_{C u}=C_{C u}^{b u l k}
$$

The surface coverage terms are constrained by time dependent boundary conditions that change as molecules are adsorbed and/or replaced at the mouth of the channel. While these rates are similar to Eq. 2 and 4 , the concentrations of inhibitor and accelerator are equal to the initial bulk concentration because of the absence of diffusion limitations at the mouth of the channel. They can be expressed as

$$
\begin{aligned}
& \text { At } z=0 \text { and } z=L \\
& \qquad \frac{\partial \theta_{I}}{\partial t}=\frac{k_{I} C_{I}^{\text {bulk }}}{\Gamma_{I}}\left(1-\theta_{I}-\theta_{A}\right)-\frac{k_{r e p} C_{A}^{\text {bulk }}}{\Gamma_{I}}\left(\theta_{I}\right) \\
& \quad \frac{\partial \theta_{A}}{\partial t}=\frac{k_{A} C_{A}^{\text {bulk }}}{\Gamma_{A}}\left(1-\theta_{I}-\theta_{A}\right)+\frac{k_{r e p} C_{A}^{\text {bulk }}}{\Gamma_{I}}\left(\theta_{I}\right)
\end{aligned}
$$

Solving Eqs. 10 and 11 simultaneously provides the boundary conditions for $\theta_{I}$ and $\theta_{A}$. Finally, initial conditions are described below in Eqs. 12 and 13:

$$
\text { At } t=0 \quad C_{I}=C_{I}^{b u l k}, \quad C_{A}=C_{A}^{b u l k}, \quad C_{C u}=C_{C u}^{b u l k}
$$

$$
\theta_{I}=0, \quad \theta_{A}=0
$$

The boundary and initial conditions have been defined such that time begins immediately after the substrate is immersed in the electrolyte bath, before any reaction takes place. Thus, appropriate conditions may be established without knowledge of the plating system. While Akolkar and Landau allowed for specific additive evolution prior to $t>0$ to simplify the boundary and initial conditions, these assumptions limit the model to a certain range of additive concentrations and sample geometries over which the simplifications are accurate. ${ }^{19}$ The state of the system immediately after submersion is determined by the additive behavior inside the channel, which is dictated by the sample geometry and electrolyte bath.

\section{Deposition Kinetics}

As explained above, the copper is deposited on the substrate according to Faraday's law (Eq. 5). The solution to the above system of equations gives a time- and location-dependent solution for the additive surface coverage in the channel. This information can then be used to simulate copper growth according to the given additive state at each site. Because a copper ion can experience three plating options, each with a unique, concentration-dependent exchange current density and cathodic transfer coefficient, the total current density will be a sum of three contributions weighted by the fractional surface coverage. Therefore, the total current density is

$$
\begin{aligned}
i= & m_{I} C_{C u} \theta_{I} \exp \left[\frac{-\alpha_{I} F \eta}{R_{g} T}\right]+m_{A} C_{C u} \theta_{A} \exp \left[\frac{-\alpha_{A} F \eta}{R_{g} T}\right] \\
& +m_{\text {free }} C_{C u}\left(1-\theta_{I}-\theta_{A}\right) \exp \left[\frac{-\alpha_{\text {free }} F \eta}{R_{g} T}\right]
\end{aligned}
$$


where the contributions from inhibitor occupied sites, accelerator occupied sites, and free sites are represented by the first, second, and third summed terms, respectively. The total time and location-dependent current density experienced by the substrate allows for a quantitative simulation of channel fill. It is evident from Eq. 14 that the local current density, and therefore the local growth rate, is directly related to the additives surface coverage distribution. This deposited growth results in a shrinking channel radius and is reflected as the time and position dependent $R$ term in Eq. 1, 3, and 8 .

\section{Model Parameters and Methods}

The plating model, described by the rate relations (Eqs. 1-4 and 8), boundary conditions (Eqs. 9-11), initial conditions (Eqs. 12-13), and ultimately deposition simulations (Eqs. 5 and 14), requires knowledge of the sample geometry $(R$ and $L)$, electrolyte composition $\left(C_{I}^{\text {bulk }}\right.$ $C_{A}^{\text {bulk }}$, and $\left.C_{C u}^{\text {bulk }}\right)$, additive properties $(k, D$, and $\Gamma)$, and deposition parameters $\left(i_{o}\right.$ and $\left.\alpha\right)$. Table I lists the values for all parameters implemented in the model, along with experimentally applied conditions that are used to study electroplating in macro-sized channels. Note the vastly different sample geometry lengths as compared to microvia filling, where $R$ and $L$ are typically in the range of $1-10 \mu \mathrm{m}$. A stiff, ordinary differential equation solver in Matlab (MathWorks, Inc.) was used to simultaneously solve the rate equations in the model. The axial dimension was discretized into 256 steps and plating was simulated for 66 hours unless otherwise noted. The radius was adjusted accordingly for plating during this time.

\section{Results}

The simple, predictive, multi-component model developed in the previous section can be implemented on any high aspect ratio channel over a wide range of length scales. A representative channel with dimensions analogous to the late-growth pores in biomorphic graphite derived from red oak (seen in Figure 1) will be used to characterize the results. In previous experimental work on this material, $1.2 \times 1.2 \times$ $0.3 \mathrm{~cm}^{3}$ scaffolds were used for plating, with the axes of the extended parallel pore channels aligned along the thinnest dimension $(\mathrm{L}=$ $0.3 \mathrm{~cm}, \mathrm{R}=0.003 \mathrm{~cm}, \mathrm{AR}=50) .{ }^{9,21}$ The resulting channels in the scaffold from the vascular pore network are assumed to be uniformly smooth and cylindrical for modeling purposes, and natural variations and small radial openings are neglected. Plating is simulated for 66 hours using a constant current of $10 \mathrm{~mA}$, which is equivalent to an activation overpotential of roughly $0.19 \mathrm{~V}$.

In order to discuss the model's capabilities and the plating trends in macro-sized channels, the bulk concentrations of the inhibitor and accelerator have been varied over a range of magnitudes, the results of which are summarized in Figure 5. The dotted lines show the

\section{Inhibitor Concentration (ppm)}
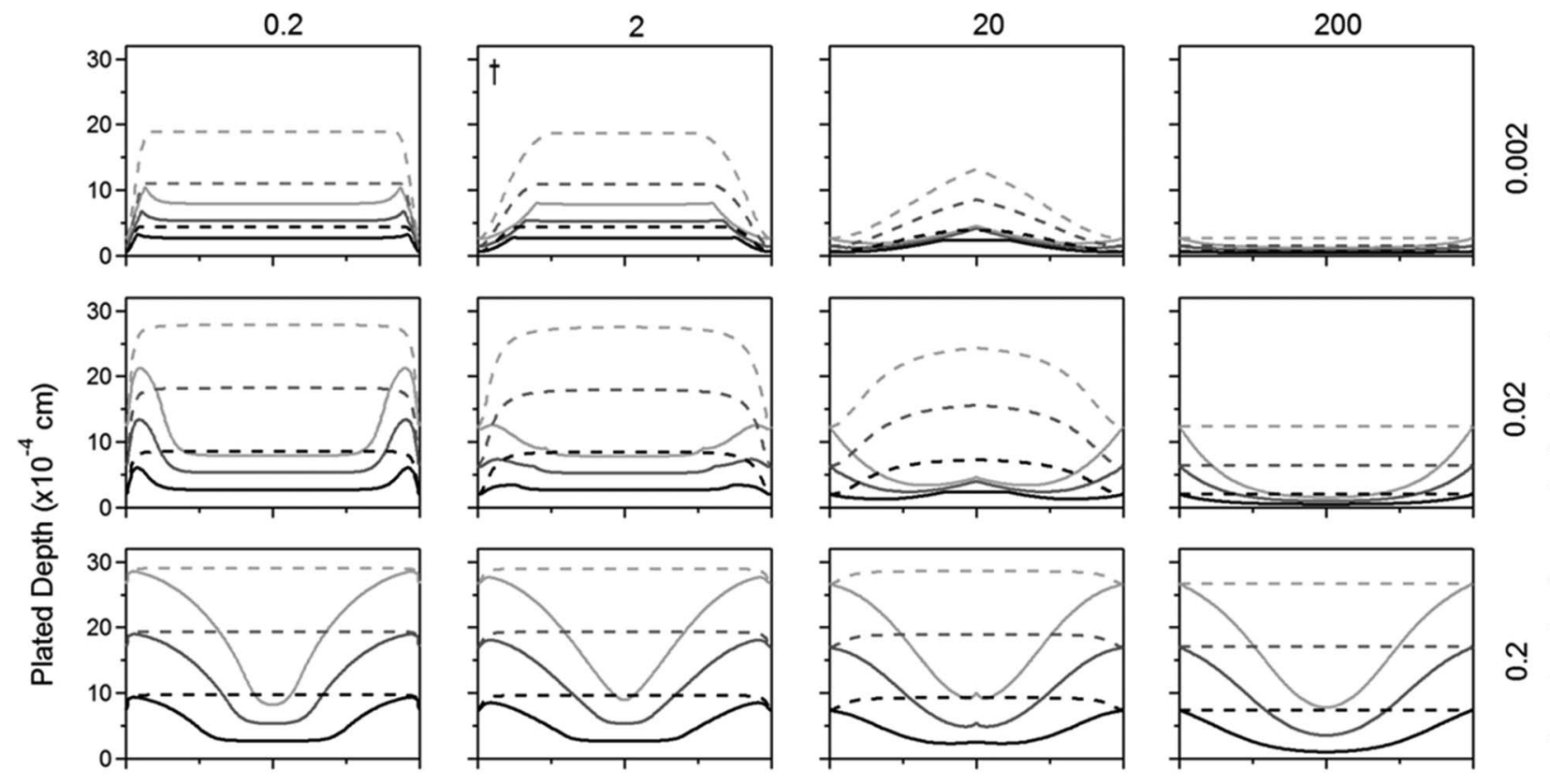

ํ.
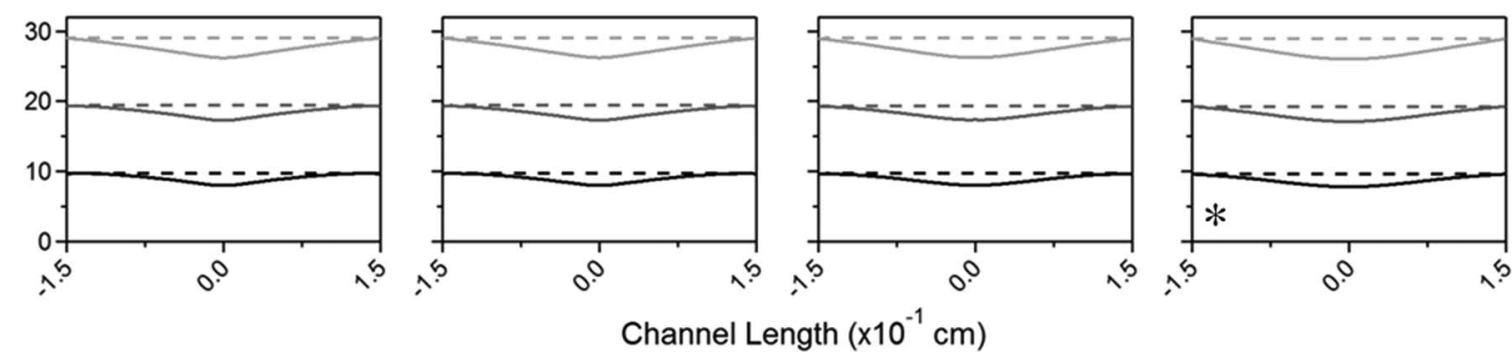

N
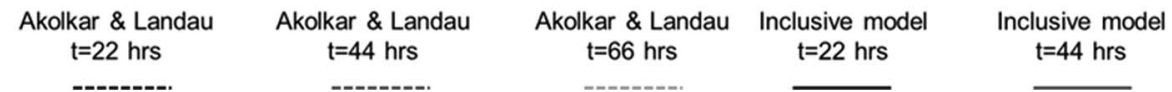

Inclusive mode $\mathrm{t}=66 \mathrm{hrs}$

Figure 5. Predicted plated depth of the copper growth on the channel walls is plotted at various times $(22,44$, and 66 hours) over the entire length of the channel (z-dimension). Plating was simulated for various inhibitor and accelerator bath concentrations in a $0.3 \mathrm{~cm}$ long channel with $0.006 \mathrm{~cm}$ diameter. Results predicted by the original model proposed by Akolkar and Landau, ${ }^{19}$ applied to this channel, are shown as dotted lines, while data from the inclusive model outlined in this paper are shown as solid lines. 


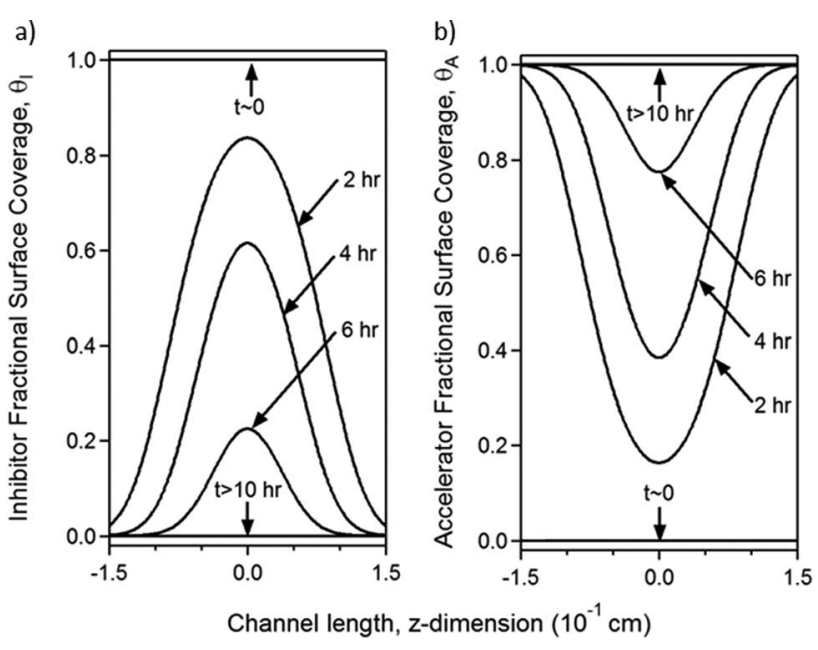

Figure 6. Fractional surface coverage of the (a) inhibitor and (b) accelerator through plating for a bath composed of $200 \mathrm{ppm}$ inhibitor and $2 \mathrm{ppm}$ accelerator. There is an initial complete coverage by the inhibitor, which is followed by a quick transition to complete accelerator coverage.

predictions from Akolkar and Landau's approach (Figure 4a), while the thick, solid lines are the results of the comprehensive model developed in this paper (Figure 4b). The latter clearly shows that the enhanced model is capable of predicting sub-conformal growth when appropriate. The range of additive concentrations shown represent the regions where plating is sensitive to additive concentration; concentrations higher than $200 \mathrm{ppm}$ inhibitor and $2 \mathrm{ppm}$ accelerator produce essentially the same results as these bounds, for reasons discussed in full below. Two specific cases of plating in macro-sized channels will be examined in this work: a bath with $200 \mathrm{ppm}$ inhibitor and 2 ppm accelerator (indicated with a $*$ in Figure 5) - the bath closest to the industry standard of roughly $200 \mathrm{ppm}$ inhibitor and $60 \mathrm{ppm}$ accelerator used in plating metal interconnects, and a bath with $2 \mathrm{ppm}$ inhibitor and $0.002 \mathrm{ppm}$ accelerator (indicated with a $\dagger$ in Figure 5) the optimal conditions of those modeled.

Figure 6 depicts the evolution of additive surface coverage on the walls of a macro-sized channel when using a standard industry bath of $200 \mathrm{ppm}$ inhibitor and $2 \mathrm{ppm}$ accelerator. Note the immediate coverage by the inhibitor, indicating that high inhibitor availability precludes a concentration gradient of inhibitor from forming. However, the accelerator is depleted as it gradually replaces the inhibitor adsorbed to the channel walls, requiring transport of accelerator molecules from the channel mouth to the center. The accelerator continues to replace the inhibitor as it diffuses toward the center. This establishes an unfavorable distribution in the channel. In this system, there is higher accelerator coverage near the mouth, whereas the mouth would be highly inhibited in an ideal system. A concave, sub-conformal copper growth profile is established, seen in the corresponding graph in Figure 5, which, once the inhibitor has been completely replaced, is maintained for the duration of plating until the channel mouth is obstructed.

Figure 7 depicts the evolution of the additive surface coverage on the walls of the channel under optimal conditions, as predicted by the model, in a bath composed of $2 \mathrm{ppm}$ inhibitor and $0.002 \mathrm{ppm}$ accelerator. An inhibitor concentration gradient is established, resulting in differential coverage between the center and mouth of the channel. This results from the initial depletion and slow diffusion that are the results of a lower bulk inhibitor concentration. The inhibitor molecules do not reach the center throughout the entire plating time. Therefore, in this scenario, the outer ends of the channel have slower plating kinetics than the middle of the channel, where free sites are still available, and differential deposition is established. It is important to note that the accelerator serves almost no function in this additive system. The favorable plating gradient is established solely between

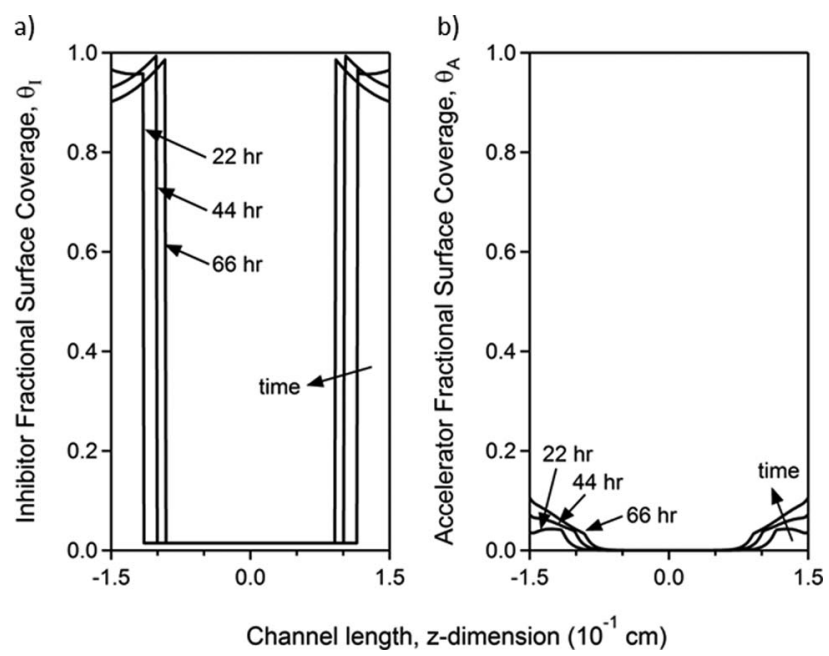

Figure 7. Fractional surface coverage of the a) inhibitor and b) accelerator through plating for a bath composed of $2 \mathrm{ppm}$ inhibitor and $0.002 \mathrm{ppm}$ accelerator. This bath provided the best plating results in the studied sample space. Due to diffusion limitations and consumptive adsorption, the inhibitor spends the entirety of the plating time in transit to the center of the channel, resulting in increased inhibitor surface coverage at the outer ends of the channel. Evan after 66 hours of plating, there is only a minimal level of accelerator surface coverage.

the inhibited channel opening and the uninhibited channel middle, as opposed to accelerated plating at the channel middle; the accelerator concentration is too low to adsorb at an appreciable rate. Experimental optimization studies of copper-electroplated biomorphic graphite have demonstrated that a measureable improvement in extent and distribution of copper infiltration is possible. This was reflected by a $72 \%$ increase in thermal conductivity of the optimized composite over the non-optimized composite with equivalent copper volume fractions. ${ }^{9,21}$

Finally, Figures 8 and 9 highlight the additions to Akolkar and Landau's model included in the current work by depicting the depletion of the accelerator and copper ions in the channel. ${ }^{19}$ Figure 8 demonstrates the need for a diffusion-limited term when describing the possible evolution of the accelerator species. This trend proves that on this length scale, assuming a constant bulk concentration, and therefore consistent availability of the accelerator for all times and at all positions, results in inaccurate predictions. Similarly, Figure 9

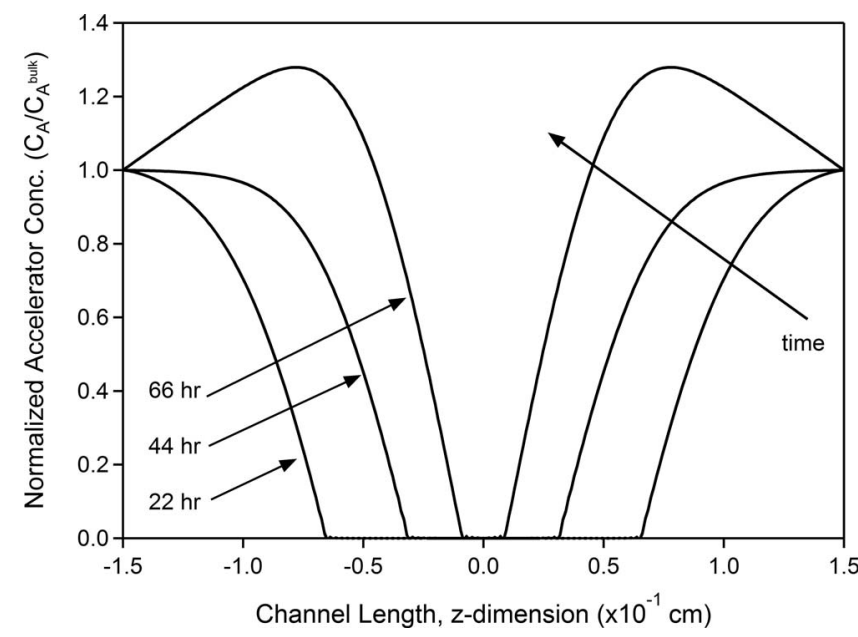

Figure 8. Accelerator concentration in the bath within the channel with respect to channel position, in a bath composed for $2 \mathrm{ppm}$ inhibitor and $0.2 \mathrm{ppm}$ accelerator. After only 22 hours of plating, the concentration of the accelerator is completely depleted in the center of the channel. 




Figure 9. Copper-ion concentration in the bath within the channel with respect to channel position, in a bath composed for $200 \mathrm{ppm}$ inhibitor and $2 \mathrm{ppm}$ accelerator. Under these conditions, after 66 hours of plating, the availability of the copper ions in the bath at the center of the channel has been depleted to roughly $87 \%$ of the bulk concentration.

justifies the inclusion of the copper ions as an additional species in the model. Due to transport limitations and consumptive plating of the ions, it is possible not only for there to be a disparity in copper ion availability, but also for plating to occur at inconsistent rates due to the reactant concentration dependence of deposition kinetics.

\section{Discussion}

Resulting plating regimes.- Akolkar and Landau defined two regimes for additive-assisted plating necessary for successful infiltration of a micro-via. ${ }^{19}$ The first is the transport-adsorption regime, which occurs during the first 5 seconds of a total 40 -second plating time for micro-sized channels. It is characterized by a period of additive movement that establishes the initial surface coverage. During this time, inhibitor coverage is initially limited to the outer ends of the channel and only trivial levels of inhibitor reach the center. As time progresses in this regime, all free sites become occupied by an additive and a differential distribution of additive coverage is established. Due to the shorter time required to plate micro-channels, a nontrivial portion of plating can be accomplished under these favorable conditions and a conformal growth profile is set up. The second phase is the additive interaction regime and is distinguished by the eventual replacement of any bonded inhibitor with accelerator. This second regime is vital when plating blind-holes to quickly plate the bottom of the trench and move the plating front outward by curvature enhanced accelerator coverage (CEAC). ${ }^{29,30,39}$ This effect occurs when there is an accumulation of accelerator near the channel's bottom due to decreased surface area as the region is plated. The accelerator/inhibitor replacement reaction occurs in a constructive manner from the inside out.

The results for macro-sized samples, however, have overlapping time scales, making it difficult to identify these regimes unambiguously. For example, for a few of the plating conditions, a constructive differential distribution of additives may be initially established. However, compared to the total time required to fill the channel, only a trivial amount of plating can occur under these favorable conditions before the distribution is disrupted as the accelerator replaces the inhibitor. In a macro-sized through-hole, there is no trench bottom, and the radius is too large for a pinched "butterfly" growth to occur. Therefore, while CEAC effects may still be present due to the steady plating of the channel walls, this investigation does not consider them for two reasons. First, the area reduction is not driven by curvature effects, and therefore the contribution to the resulting plating profile will be modest compared to a blind-hole. Second, by neglecting CEAC con- tributions, it is possible to isolate additive transport and adsorption effects that arise specifically in macro-channels. Consequently, after the ideal coverage is disrupted, the plating is dictated by the transport limited accelerator, which often creates an unfavorable distribution in macro-sized channels. This highlights the need to establish conformal growth in the first stage, which has shown to be difficult due to the long plating times required. Therefore, at this macro-length scale, it is not possible to establish a constructive distribution between the accelerator and inhibitor using any combination of initial additive concentrations in the electrolyte bath. Better results would be obtained in this scaffold by using a simple one-additive electrolyte system, where a favorable distribution could be established and maintained between the slow diffusing inhibitor at the mouth of the channel, and uninhibited plating at the middle. By eliminating the accelerator, the detrimental effects of its transport limitations and the quickly dominating additive interaction regime would be removed. This result is in agreement with previous studies on micro-via systems that have concluded that through-holes with parallel walls are filled more completely by a one additive system. ${ }^{45}$

The results shown here have been established for an applied overpotential of $0.19 \mathrm{~V}$. Nominally, this parameter is an easy way to adjust the plating rate of the system by raising or lowering the total current density experienced at the substrate surface. While adjustments to this value may also slightly alter the predicted plating profile, the investigation shows that its effects are insignificant when compared to the effects of the additive concentrations and characteristics.

The difficulty in infiltrating a channel of this size with the given bath demonstrates how additive systems designed to promote conformal plating must exhibit a balance between several competing rates. The precise timing of species depletion, diffusion, adsorption, plating, and replenishment is vital to achieving both the desired surface coverage of the additives and void-free copper filling. The success of this system is therefore dictated by individual species characteristics, as well as the sample geometry. Other studies have attempted to characterize the trend between additive behavior and sample geometry with non-dimensional relations, such as aspect ratio. ${ }^{19,31,46,47}$ While these trends are useful in comparing results on similar length scales, consideration of the actual channel dimensions is equally important. As an example, the effectiveness of this inhibitor/accelerator system in macro-sized channels, represented here by porous biomorphic graphite, can be assessed in terms of the comparably large axial and radial dimensions as compared to micro-sized channels.

Axial dimension.- Figures 8 and 9 show that the accelerator and copper ions are consequentially transport-limited at the macro- length scale despite their faster diffusion rates. This can be detrimental to the infiltration process for several reasons. In an ideal system, the purpose of the accelerator is to reach the middle of the channel first, adsorbing to free sites to reserve the inner region for rapid plating before the slower moving inhibitor reaches the area. In the microsized systems it was designed for, it can be assumed that the effects of the transport limitations on the accelerator are trivial, and it is therefore never depleted below its bulk concentration, allowing this favorable distribution to be established and maintained. A significant fraction of the total plating can occur under ideal differential coverage before the stronger bonding accelerator eventually displaces the inhibiting film at the opening to the channel. However, for most common accelerator concentrations, it is quickly depleted in channels of this length due to the larger interior surface area and must be replenished from the bulk electrolyte through the channel opening. Describing diffusion speed as qualitatively "fast" or "slow" is relative to the distance over which the molecules must travel, and at greater length scales, while it does diffuse faster than inhibitor molecules, the effects of the transport limitations on the accelerator are evident. Therefore, the previously defined relationship of "fast" diffusion and "slow" adsorption of the accelerator necessary for conformal growth is no longer maintained, as the accelerator begins the replacement reaction before even reaching the center of the channel. The balance between accelerator velocity 
and adsorption rate is lost because these processes are no longer functioning on the same time scale. Therefore, it is difficult to establish a favorable distribution for times sufficient to produce significant plating. However, as can be seen in Figure 5 by moving from lower to higher accelerator concentrations at constant inhibitor concentrations, if increased diffusion speed is attempted by increasing the gradient between the electrolyte at the channel mouth and channel center (the result of a higher initial accelerator concentration), the driving force for additive adsorption is also enhanced - both in the center, where it is desired, and near the mouth, where it is not. The complete replacement of inhibitor by accelerator simply occurs faster.

Secondly, while less pronounced in the plating conditions used in the current examination, depletion of the copper ions in solution is also an obstacle. Because plating rate is directly proportional to copper ion concentration, if a concentration gradient of copper ions exists between the depleted channel center and easily replenished area near the channel opening, the copper will plate faster near the mouth of the channel, resulting in choke-off. This problem is exacerbated when the outer ends of the channel have a higher coverage of accelerator, as the exchange current density associated with an accelerator occupied site is the most sensitive to copper ion concentration, as seen in Table I. The combined effects of unfavorable additive distributions and higher copper ion concentrations near the channel opening contribute to the difficulty in plating high aspect ratio channels of large length scale. Electrolyte baths utilizing high copper concentrations and low acidity, such as the bath used in this study, reduce this effect by promoting faster diffusion and delayed depletion of the ions.

Given the species diffusivity parameters and limitations, the axial dimension of a channel effectively dictates the lower boundary of useful additive concentrations. For the inhibitor, a concentration gradient is vital to establishing the correct differential coverage of additives. The axial dimension determines the range over which the additive transport occurs on the same time scale as the required plating time. However, because a higher coverage of the accelerator species is desired at the center of the channel, a concentration gradient of the species between the mouth and this location is detrimental to ideal differential coverage. Eventually, at low additive concentrations, the diffusion of the species is so slow over the length of the channel that it renders the additive ineffective, and plating proceeds essentially as if it were not present.

Radial dimension.- Increased radial dimensions in a channel also play a role in preventing the establishment of a constructive additive distribution. At the opening to the channel, the radial plane can be thought of as a gate that permits bath species to enter the pore. Because the area associated with this flux scales to the square of the channel radius, it becomes challenging to make small adjustments to the influx of additive species as the size of the radius increases from micro- to macro-sized. The goal is to maintain a steady stream of favorably diffusing/interacting additives within the channel without flushing the system. However, with a large supply of molecules due to a large channel entrance, it becomes difficult to achieve this goal without reducing the initial, bulk concentrations of the species to impractically low values. The significance of this issue is readily observed by examining the initial conditions of a given system. For example, at $200 \mathrm{ppm}$ inhibitor, the amount of inhibitor available in a via-sized $(\mathrm{R}=0.1 \mu \mathrm{m}, \mathrm{L}=1 \mu \mathrm{m})$ volume of electrolyte initially present in the channel after it is submerged is two orders of magnitude less than the amount required to completely saturate the inner surface area of the channel. The additive system was designed in this manner for micro-sized channels to initially deplete inhibitor concentration, thus establishing a concentration gradient along the length of the channel and driving the inhibitor in from the channel ends. However, in a macro-sized channel volume of electrolyte containing $200 \mathrm{ppm}$ inhibitor, there is roughly 1.5 times more inhibitor than the amount needed to coat the entire inner wall available in the initial volume. Consequently, there will be an immediate saturation of inhibitor coverage on the entire surface due to its quicker adsorption compared to the other species, rendering the remainder of the plating time and possible additive evolution ineffective, as seen in the sub-conformal growth depicted in Figure 5 for 200 ppm inhibitor concentrations. Suitable initial coverage of the additives is a key part in establishing the favorable differential coverage and is dictated in a large part by the magnitude of the channel radius. Based on the geometry of the late-growth channels in a biomorphic scaffold produced from red oak, complete initial saturation occurs at concentrations greater than $135 \mathrm{ppm}$. This result highlights the relationship between sample geometry and additive function. The behavior of an additive is dictated by the channel dimensions, and an effective bath composition is unique to a specific channel size - there are no blanket solutions. This makes infiltrating scaffolds that have bimodal pore size distributions, such as biomorphic graphite produced from red oak, very difficult, as the additive system cannot be optimized for both size scales simultaneously.

The effects of high concentrations of accelerator allowed into the channel, possibly by an increased radial dimension, have been discussed in the previous section - the elevated availability of accelerator causes the replacement reaction to occur very quickly, resulting in non-optimized plating profiles. According to this study, and shown in Figure 5, accelerator concentrations should be kept below $1 \mathrm{ppm}$ to avoid completely dominating the macro-sized systems of this scale. However, the range of useful accelerator concentration quickly dips in to uncontrollably low concentrations, and therefore, in these baths, it is essentially no different than using a single-additive system.

In principle, the radial dimension of the channel determines the upper boundary of effective additive concentrations. At high concentrations, the amount of additives permitted into the channel is too large to fine tune, and the nuances of the additive characteristics that contribute to conformal growth cannot be observed. This can result in adverse initial conditions, as with the fast adsorbing inhibitor molecules, or a dominating species, as with the stronger and irreplaceably bonding accelerator molecules.

Additionally, because the copper is plated on the walls of the channel and grows inward, the radial dimension determines the amount of time required to fill the channel according to the plating rate outlined in Eq. 9. Increased plating times require that the delicate differential coverage of the two additives is maintained for a longer time.

Applications of two-additive systems.- While other work has studied the plating of copper in through holes on micro-scales with a one-additive electrolyte, two-additive bath are still advantageous if the system can maintain a correct balance between diffusion, adsorption, replacement, and plating. ${ }^{45,48}$ Obviously this will depend on the specific additives used and the geometry of the scaffold. This paper has shown that simplified rules which only consider channel aspect ratio are not useful over all size scales. The magnitude of the channel dimensions, and their relation to the characteristics of the additives, is what determines the additive distribution in a two-additive system, and a ratio of the dimensions obscures this nuance.

In an attempt to investigate the use of electroplating as a means of infiltration in various porous scaffolds, the model was used to simulate plating in channels over dimensions ranging $1 \mu \mathrm{m}$ to $2.5 \mathrm{~mm}$ and resulting aspect ratios from 1 to 80 . Combinations of inhibitor and accelerator concentrations were sought in which it was possible to maintain a constructive differential coverage of additives. Figure 10 depicts the results of this study, in which many orders of magnitude of length have been investigated over a range of aspect ratios. The area in which successful plating is expected due to additive-assisted plating of two species can be approximated by the relationship $A R<\frac{0.7}{L}$. Note that this relation is not evidence of an intrinsic property of the plating system, but offered simply as a convenient guide. Once it has been determined that an optimized solution is possible on the given length scale for a two-additive bath, the general trends relating to aspect ratio, outlined by others, can be used, along with the provided model, to fine tune the system. 




Figure 10. Channels with varying dimensions were tested in the model and the results are classified according to three possible outcomes. Based on these results, the dark gray highlighted area indicates the sample space in which a two-additive electrolyte bath composed of both an accelerator and inhibitor can result in void-free plating in a through-hole. Samples with dimensions that fall in the white area will not benefit from this type of bath.

\section{Conclusions}

Additive-assisted copper electroplating in macro-channels has been investigated using a multi-element, time-dependent model to simulate growth. The model, produced by building upon the work of Akolkar and Landau, is independent of sample geometry and initial additive concentrations. The model relies on a robust description of all possible movement of bath species within the channel due to diffusion, adsorption, replacement, or plating. These additions to the model were necessary to provide accurate results at larger feature sizes. Comparisons of the Akolkar and Landau model with the model outlined in this paper show that predictions of sub-conformal growth in these macro-channels is now possible. Based on a study of the effectiveness of the inhibitor/accelerator additive system, it is clear that bath compositions used for interconnect metallization are not appropriate for use in these larger channels. In fact, conformal growth is shown to be quite difficult to achieve on the length scales seen in biomorphic graphite scaffolds using a two-additive system due to the delicate balance required between the rates of species diffusion, adsorption, and plating within the channel. Establishing a sustained differential additive coverage scenario between the inhibitor and accelerator is not possible in this system, and the best results were observed simply between the differential coverage between the inhibitor and free sites on the surface. While relationships utilizing only the channel aspect ratio proved to be oversimplified, the model was used to develop a straightforward connection between aspect ratio and channel length at the given plating conditions, as well as explain the observed trends involving channel dimensions. This guide is meant to facilitate the processing of multicomponent materials, but a full analysis using the comprehensive model is suggested given the systems specific characteristics (feature size, plating conditions, additive type, etc.), which can result in the establishment of optimized conditions.

\section{Acknowledgments}

This work was supported by the National Science Foundation, DMR-0710630. SEM work was performed in the EPIC facility of the NUANCE Center at Northwestern University. NUANCE Center is supported by NSF-NSEC, NSF-MRSEC, The Keck Foundation, The State of Illinois, and Northwestern University. Helpful discussions with Professor David Chopp at Northwestern University are acknowledged.

\section{List of Symbols}

$A R$ aspect ratio (dimensionless)

$\alpha \quad$ cathodic transfer coefficient $\left(\mathrm{mol}^{-1}\right)$

C concentration $\left(\mathrm{mol} / \mathrm{cm}^{3}\right)$

$\Gamma$ saturation surface concentration $\left(\mathrm{mol} / \mathrm{cm}^{2}\right)$

$D$ diffusion coefficient $\left(\mathrm{cm}^{2} / \mathrm{s}\right)$

$F \quad$ Faraday's constant $(\mathrm{C} / \mathrm{mol})$

$h$ plated thickness $(\mathrm{cm})$

$\eta \quad$ overpotential (V)

$\theta$ fractional surface coverage (dimensionless)

$i$ current density $\left(\mathrm{A} / \mathrm{cm}^{2}\right)$

$i_{0} \quad$ exchange current density $\left(\mathrm{A} / \mathrm{cm}^{2}\right)$

$J$ flux of species in solution $\left(\mathrm{mol} / \mathrm{cm}^{2} \mathrm{~s}\right)$

$k$ adsorption rate constant $(\mathrm{cm} / \mathrm{s})$

$L$ channel length (cm)

$m$ relational constant between $i$ and $C(\mathrm{~A} \cdot \mathrm{cm} / \mathrm{mol})$

$n$ electrons transferred (dimensionless)

$R$ channel radius $(\mathrm{cm})$

$R_{g} \quad$ universal gas constant $(\mathrm{J} / \mathrm{mol} \cdot \mathrm{K})$

$\rho$ density $\left(\mathrm{g} / \mathrm{cm}^{3}\right)$

$t$ time (s)

$T$ temperature $(\mathrm{K})$

$z \quad$ distance coordinate $(\mathrm{cm})$

\section{Subscripts}

$A$ related to the accelerator molecule

$\mathrm{Cu}$ related to the copper ions

I related to the inhibitor molecule

rep related to the replacement reaction

tot total value

\section{Superscripts}

A occurring on an accelerator occupied site

ads related to adsorption

bulk bulk value

diff related to diffusion

free occurring on an unoccupied site

I occurring on an inhibitor occupied site

\section{References}

1. M. Schlesing and M. Paunovic, Modern Electroplating, Wiley, New York (2000).

2. U. Cohen, F. B. Koch, and R. Sard, J. Electrochem. Soc., 130, 1987 (1983).

3. P. C. Andricacos and N. Robertson, IBM J. Res. Dev., 42, 671 (1998).

4. P. C. Andricacos, C. Uzoh, J. O. Dukovic, J. Horkans, and H. Deligianni, IBM J. Res. Dev., 42, 567 (1998)

5. L. Economikos, X. Wang, A. Sakamoto, P. Ong, M. Naujok, R. Knarr, L. Chen, Y. Moon, S. Neo, J. Salfelder, A. Duboust, A. Manens, W. Lu, S. Shrauti, F. Liu, S. Tsai, and W. Swart, Proc. IEEE Int. Interconnect Technology Conf., 233 (2004).

6. Z. Y. Wang, L. W. Wang, N. T. Nguyen, W. A. H. Wien, H. Schellevis, P. M. Sarro, and J. N. Burghartz, IEEE Trans. Adv. Packag., 29, 615 (2006).

7. R. H. Havemann and J. A. Hutchby, Proc. IEEE, 89, 586 (2001)

8. K. E. Pappacena, M. T. Johnson, S. Xie, and K. T. Faber, Compos. Sci. Technol., 70, 485 (2010).

9. M. T. Johnson, A. S. Childers, J. Ramirez-Rico, H. Wang, and K. T. Faber, Composites Part A, 53, 182 (2013).

10. A. R. D. Lopez, J. M. Fernandez, F. M. V. Feria, R. E. Sepulveda, M. J. L. Robledo, J. Llorca, J. Y. Pastor, M. Presas, K. T. Faber, V. S. Kaul, K. E. Pappacena, and T. E. Wilkes, Ceram. Eng. Sci. Proc., 27, 635 (2007).

11. K. E. Pappacena, S. P. Gentry, T. E. Wilkes, M. T. Johnson, S. Xie, A. Davis, and K. T. Faber, J. Eur. Ceram. Soc., 29, 3069 (2009).

12. V. S. Kaul, K. T. Faber, R. Sepulveda, A. R. D. Lopez, and J. Martinez-Fernandez, Mater. Sci. Eng. A-Struct. Mater. Prop. Microstruct. Process., 428, 225 (2006).

13. A. Herzog, U. F. Vogt, S. Siegmann, and O. Beffort, Adv. Eng. Mater., 8, 980 (2006)

14. P. Greil, T. Lifka, and A. Kaindl, J. Eur. Ceram. Soc., 18, 1961 (1998). 
15. H. Sieber, C. Hoffmann, A. Kaindl, and P. Greil, Adv. Eng. Mater., 2, 105 (2000).

16. L. J. Gibson and M. F. Ashby, Cellular Solids: Structure and Properties, Cambridge University Press, New York (1999)

17. J. W. Gallaway, M. J. Willey, and A. C. West, J. Electrochem. Soc., 156, D146 (2009).

18. M. A. Pasquale, L. M. Gassa, and A. J. Arvia, Electrochim. Acta, 53, 5891 (2008).

19. R. Akolkar and U. Landau, J. Electrochem. Soc., 156, D351 (2009).

20. P. M. Vereecken, R. A. Binstead, H. Deligianni, and P. C. Andricacos, IBM J. Res. Dev., 49, 3 (2005).

21. M. T. Johnson, A. S. Childers, F. De Carlo, X. Xiao, and K. T. Faber, Compos. Sci. Technol. (In review).

22. A. C. West, J. Electrochem. Soc., 147, 227 (2000).

23. A. C. West, C. C. Cheng, and B. C. Baker, J. Electrochem. Soc., 145, 3070 (1998).

24. K. M. Takahashi and M. E. Gross, J. Electrochem. Soc., 146, 4499 (1999).

25. C. Madore, M. Matlosz, and D. Landolt, J. Electrochem. Soc., 143, 3927 (1996).

26. C. Madore and D. Landolt, J. Electrochem. Soc., 143, 3936 (1996).

27. Y. Cao, P. Taephaisitphongse, R. Chalupa, and A. C. West, J.Electrochem. Soc., 148 C466 (2001).

28. M. Georgiadou, D. Veyret, R. L. Sani, and R. C. Alkire, J. Electrochem. Soc., 148 C54 (2001)

29. D. Josell, D. Wheeler, W. H. Huber, J. E. Bonevich, and T. P. Moffat, J. Electrochem. Soc., 148, C767 (2001).

30. A. C. West, S. Mayer, and J. Reid, Electrochem. Solid State Lett., 4, C50 (2001).

31. S. H. Chan and H. Y. Cheh, J. Appl. Electrochem., 31, 605 (2001).

32. J.-W. E. Chern and H. Y. Cheh, J. Electrochem. Soc., 143, 3144 (1996).

33. D. Josell, D. Wheeler, and T. P. Moffat, J. Electrochem. Soc., 159, D570 (2012).

34. A. Pohjoranta and R. Tenno, J. Electrochem. Soc., 154, D502 (2007).
35. T. Matsuoka, K. Otsubo, Y. Onishi, K. Amaya, and M. Hayase, Electrochim. Acta, 82, 356 (2012).

36. T. P. Moffat, D. Wheeler, and D. Josell, J. Electrochem. Soc., 151, C262 (2004).

37. D. Stoychev and C. Tsvetanov, J. Appl. Electrochem., 26, 741 (1996).

38. Z. V. Feng, X. Li, and A. A. Gewirth, J. Phys. Chem. B, 107, 9415 (2003).

39. T. P. Moffat, D. Wheeler, W. H. Huber, and D. Josell, Electrochem. Solid-State Lett., 4, C26 (2001)

40. T. P. Moffat, D. Wheeler, M. D. Edelstein, and D. Josell, IBM J. Res. Dev., 49, 19 (2005).

41. R. Akolkar and U. Landau, J. Electrochem. Soc., 151, C702 (2004).

42. K. R. Hebert, J. Electrochem. Soc., 148, C726 (2001).

43. V. M. Dubin, R. Brewer, H. Simka, and S. Shankar, Future Fab. Int., 13, 244 (2002).

44. M. S. Moats, J. B. Hiskey, and D. W. Collins, Hydrometallurgy, 56, 255 (2000).

45. W.-P. Dow, D.-H. Liu, C.-W. Lu, C.-H. Chen, J.-J. Yan, and S.-M. Huang, Electrochem. Solid-State Lett., 14, D13 (2011).

46. T. P. Moffat, J. E. Bonevich, W. H. Huber, A. Stanishevsky, D. R. Kelly, G. R. Stafford, and D. Josell, J. Electrochem. Soc., 147, 4524 (2000).

47. P. Dixit and J. M. Miao, J. Electrochem. Soc., 153, G552 (2006).

48. W.-P. Dow, H.-H. Chen, M.-Y. Yen, W.-H. Chen, K.-H. Hsu, P.-Y. Chuang, H. Ishizuka, N. Sakagawa, and R. Kimizuka, J. Electrochem. Soc., 155, D750 (2008).

49. J. P. Healy, D. Pletcher, and M. Goodenough, J. Electroanal. Chem., 338, 155 (1992).

50. J. Mendez, R. Akolkar, and U. Landau, J. Electrochem. Soc., 156, D474 (2009).

51. E. E. Farndon, F. C. Walsh, and S. A. Campbell, J. Appl. Electrochem., 25, 574 (1995).

52. A. Damjanov, T. H. V. Setty, and J. O. Bockris, J. Electrochem. Soc., 113, 429 (1966).

53. C. J. Milora, J. F. Henricks, and W. C. Hahn, J. Electrochem. Soc., 120, 488 (1973).

54. E. Mattsson and J. O. Bockris, Trans. Faraday Soc., 55, 1586 (1959). 